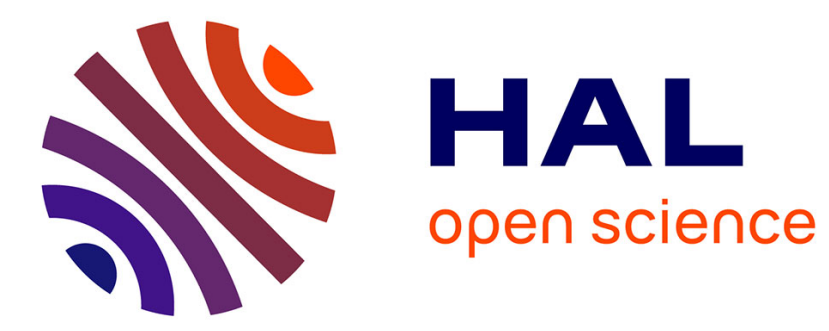

\title{
Solute tranport in periodical heterogeneous porous media: importance of observation scale and experimental sampling
}

Samer Majdalani, Jean-Philippe Chazarin, Carole Delenne, Vincent Guinot

\section{- To cite this version:}

Samer Majdalani, Jean-Philippe Chazarin, Carole Delenne, Vincent Guinot. Solute tranport in periodical heterogeneous porous media: importance of observation scale and experimental sampling. Journal of Hydrology, 2015, 520, pp.52-60. 10.1016/j.jhydrol.2014.10.065 hal-01101494

\author{
HAL Id: hal-01101494 \\ https://hal.science/hal-01101494
}

Submitted on 9 Jan 2015

HAL is a multi-disciplinary open access archive for the deposit and dissemination of scientific research documents, whether they are published or not. The documents may come from teaching and research institutions in France or abroad, or from public or private research centers.
L'archive ouverte pluridisciplinaire HAL, est destinée au dépôt et à la diffusion de documents scientifiques de niveau recherche, publiés ou non, émanant des établissements d'enseignement et de recherche français ou étrangers, des laboratoires publics ou privés. 
1 Solute transport in periodical heterogeneous porous media: Importance

S. Majdalani (1), J.P. Chazarin (2), C. Delenne (1), V. Guinot (1, 3).

(1) Université Montpellier 2 Polytech Montpellier/HSM, CC 057, Place Eugène Bataillon, 34095 Montpellier Cedex 5, France

(2) Institut de Recherche pour le Développement (IRD)/ HSM, CC 057, Place Eugène Bataillon, 34095 montpellier Cedex 5, France

(3) Inria, Team LEMon

Corresponding Author: Samer Majdalani

Tel: +33467149059

Fax : +33 467144774

Email: samer.majdalani@msem.univ-montp2.fr

Acepted for publication in Journal of Hydrology, 520, 52-60, 2015 


\section{Abstract}

This paper focuses on the effects of the observation scale and sampling on the dispersion of tracers in periodical heterogeneous porous media. A Model Heterogeneous Porous Medium (MHPM) with a high degree of heterogeneity was built. It consists of a preferential flow path surrounded by glass beads. 44 tracer experiments were carried out on several series of periodic MHPM to investigate the effect of the observation scale on solute dispersion. Each series was replicated several times, allowing for a statistical description of the unit transfer function of the MHPM. No significant trend was found for the dispersion coefficient as a function of the size of the MHPM. However, given the variability of the breakthrough curves from one experiment replicate to another, under-sampling might easily lead to conclude that the dispersion coefficient is variable with distance. Depending on the samples used, it would be as easy to (wrongly) detect an increasing trend as to detect a decreasing one. A confidence interval analysis of the experimental breakthrough curves in the Laplace space shows that (i) there exists a model with scale independent parameters that can describe the experimental breakthrough curves within the limits of experimental uncertainty, (ii) this model is not the Advection-Dispersion (AD) model, (iii) the modelling error of the $\mathrm{AD}$ model decreases with the number of periods, (iv) the size of the Reference Elementary Volume for the dispersion coefficient is between 10 and 20 periods. The effects of sampling prove to override those of scaling. This, with the invalidity of the AD model, leads to question attempts to calibrate and/or identify trends in the dispersion coefficient at intermediate scales from a limited number of experiment replicates.

\section{Keywords}

Dispersion modelling

Intermediate Scale Experiment

Heterogeneous media

\section{Introduction}

Understanding solute transport in porous media is important to predict the fate of contaminants in natural soils. Natural soils are highly heterogeneous and implicate various transport mechanisms. Tracer experiments on laboratory soil columns made a big contribution to identify these mechanisms. The most widespread transport model for inert solute is the Advection-Dispersion (AD) model. Fitting this model against real-world tracer tests has been reported to induce an increase in the dispersion coefficient with the travelled distance (see e.g. Dagan, 1989; Gelhar et al., 1992; Zhou and Selim, 2003). The variability of the dispersion coefficient with distance has motivated a strong interest for alternative models. Recently, laboratory tracer experiments have been used as a benchmarking basis to test the respective accuracy of a variety of normal and anomalous transport models (see e.g. the review in Gao et al., 2009). It has also been suggested by some authors that the dispersion coefficient in mobile-immobile models should be made exponentially dependent on distance in order to accurately reproduce experimental breakthrough curves in laboratory column experiments (Gao et al., 2010).

A number of laboratory tracer experiments in porous media are available from the literature (Silliman et al., 1998). Laboratory experiments on heterogeneous porous media are very few in comparison to those done on homogeneous porous media. One cause might be the practical difficulty to conceive and construct heterogeneous porous media.

Silliman and Simpson (1987) used fine sand inclusions (small cubes) with coarse sand surrounding. They found a change in the shape of the breakthrough curve at each of five measurement sections, and inferred a continuous increase in dispersivity with distance. Saiers et al. (1994) used a coarse sand inclusion (tubule) with finer sand surrounding. The inclusion constituted a preferential flow path and contributed to more than $60 \%$ of the mass balance. The breakthrough curves exhibited multiple inflection points that cannot be accounted for by the classical Advection Dispersion (AD) model. In comparison to other studies, the work of Saiers et al. (1994) is distinguished by the high degree of heterogeneity of the porous media. This fact is reflected in the shape of the breakthrough curves. Much 
more than a mere tailing effect, the breakthrough curves exhibited a clear two-step shape. Li et al. (1994) used coarse inclusions (polyethylene porous cylinders) with finer sand surrounding. The resulting breakthrough curves exhibited asymmetry. Huang et al. (1995) used a $12.5 \mathrm{~m}$ long column with a wide range of soil materials. Their breakthrough curves exhibited irregular and nonsigmoidal distributions and were in most cases poorly described by the AD model.

Sternberg et al. (1996) and Irwin et al. (1996) were the first to use a periodical heterogeneous porous media, where the heterogeneity consisted of a succession of several glass bead sets tested in several serial orders. The breakthrough curves were used to infer the variation of the dispersion coefficient with distance. Unfortunately, the breakthrough curves are not shown in the publications and no estimate is provided for the goodness-of-fit of the $\mathrm{AD}$ model. It is thus impossible to determine whether fitting the coefficients of the AD model was meaningful. However, both studies present the results of dispersion estimations. Sternberg et al. (1996) found that dispersion does not necessarily increase with scale, but that it can increase and decrease depending on the particular arrangement of properties in the medium. They deduced that the system length was unimportant in observing the scale effect. Conversely, in the study of Irwin et al. (1996), dispersion appeared to be scale dependent up to a certain travel distance. But, quoting the authors' own words, "because of the scatter in the data, it could be argued that the apparent increase in longitudinal dispersion is simply a result of the variation in the data". This raises the issue of sampling effects.

Greiner et al. (1997) used aggregates of lower permeability in a surrounding of higher permeability. Niehren and Kinzelbach (1998) used cylindrical cellpore filters (less permeable) in a quartzsand surrounding (more permeable). Tran Ngoc et al. (2011) used spherical clay inclusions (micro porosity) surrounded by sand (macro porosity). Danquigny et al. (2004) performed tracer tests on two kinds of heterogeneous porous material: a channel structured medium $(5.6 \mathrm{~m} \times 1 \mathrm{~m} \times 1 \mathrm{~m})$, with channels crossing the whole tank, and a statistically correlated random structure. They found that the breakthrough curves of the statistically correlated random structure could be fitted with the AD model (at least for long distances), but that the fit is quite poor for channel structured model due to the non-Gaussian distribution of the concentration.

Zin et al. (2004) designed an artificial porous medium with several conductivity contrasts, using low conductivity inclusions (small beads) surrounded by a high conductivity matrix (large beads). They found that the breakthrough curves of the low-contrast medium showed no tailing, while those of highcontrast medium showed tailing. Golfier et al. (2011) used fine sand inclusions (lenses) with coarse sand surrounding. Their breakthrough curves showed asymmetry. The low degree of heterogeneity in the studies of Sternberg et al. (1996) and Irwin et al. (1996) might have led to some contradictory conclusions concerning the dispersion scale dependence. As to the study of Saiers et al. (1994), the authors were not mainly interested in the problematic of dispersion scale dependence, but rather in colloidal mobilization and transport in a structured porous media.

In the light of the abovementioned studies, it appears that studying the effect of heterogeneity on solute transport requires two conditions: (i) a high degree of heterogeneity of the porous media (as the experimental model of Saiers et al. (1994)), and (ii) a periodical heterogeneous porous media (as the experimental protocol of Sternberg et al. (1996) and Irwin et al. (1996)). The first condition guarantees that the effects of material heterogeneity are clearly visible, while the second allows the influence of scale effects to be assessed. This also allows for a better quality of the experimental data, with easily replicable experiments yielding reliable statistics.

In this study, we conceived a Model Heterogeneous Porous Medium (MHPM) with a high degree of heterogeneity. The purpose is to (i) enrich the experimental database of laboratory tracer tests with high quality data, (ii) answer the following six questions.

(Q1) Can a scaling trend be observed for the dispersion coefficient in this highly heterogeneous MHPM?

(Q2) If this is not the case, can the variations of the dispersion with the observation scale be attributed to experimental artefacts such as sampling effects? 
(Q3) Does there exist a model with uniform coefficients (thus scale-independent) to describe adequately the present MHPM response?

(Q4) Can the AD model be considered valid at the scale of this MHPM?

(Q5) If it cannot, does a scale exist above which the $\mathrm{AD}$ model can be deemed a satisfactory approximation of the experimental response of the MHPM?

(Q6) Can more advanced models such as the Mobile-Immobile (MI) or Continuous Time Random Walk (CTRW) models be considered as better candidates than the AD model to simulate smalldistance and short-time MHPM behaviour?

The detailed characteristics of the experimental setup is described in Section 2 of the paper. The results of experimental breakthrough curves are shown in Section 3. Such experiments are usually termed Intermediate Scale Experiments (ISE) in that they involve a scale larger than the typical size of the heterogeneity but they do not achieve the size of the Reference Elementary Volume (REV) above which the continuous medium formalism should be expected to be valid (Bear, 1972).

Section 3 is also devoted to an analysis of the transfer function of the MHPM. Section 4 is devoted to discussion. A point-by-point answer to the six questions above is given throughout Section 3 and 4.

\section{Materials and methods}

\subsection{Model heterogeneous porous media}

The MHPM consists of a set of PVC columns (10 cm in diameter, $15 \mathrm{~cm}$ in length each) containing $1 \mathrm{~mm}$ glass spheres (40\% porosity) surrounding a cylindrical cavity $(2.5 \mathrm{~cm}$ in diameter, $10 \mathrm{~cm}$ in length) placed in the centre of the column (Figure 1). The cylindrical cavity has $100 \%$ porosity and thus acts as a wide pore that induces preferential water and solute transfer. The pore volume $V_{0}$ of one MHPM period is about $461 \mathrm{ml}$.

The water and solute flow enters and leaves the column by 4 inlets and outlets ( $4 \mathrm{~mm}$ in diameter). A $500 \mu \mathrm{m}$ sieve prevents the glass spheres from clogging the inlets and outlets, possibly blocking the flow. Figure 2 shows real captures of the MHPM. The incoming discharge (imposed by a peristaltic pump) is equally distributed between the 4 inlets. It is expected that the flow of the central inlet will mainly transfer through the central cavity, while the flow of the three remaining inlets will mainly transfer through the surrounding glass beads.

After carrying out the series of experiments, four randomly chosen columns were disassembled. In each of them, the position of the sieve was compared to what it was before the experiment. No detectable displacement was noted.

\subsection{Experimental setup}

Step tracing experiments are done by injecting salty water (deionised water supplemented with $\mathrm{NaCl}$ at $C_{0}=0.1 \mathrm{Mol} / \mathrm{l}$ ) in a study column initially containing deionised water. A flow of $7.5 \mathrm{l} \mathrm{h}^{-1}$ salty water is induced into the study column until the outlet concentration $C$ stabilizes to $C_{0}$. In these experiments, the stable $C_{0}$ level was generally reached after injecting three times the pore volume $V_{0}$ of the study column. The outlet concentration is measured using a conductimeter (WTW TetraCon 325) and saved on a data logger (Campbell CR1000) every $5 \mathrm{~s}$. The cumulated outlet volume $V$ is measured by weighing the effluent every $5 \mathrm{~s}$ and saved on the data logger (Figure 3).

The study column consists of a series of $N$ connected MHPM. In this study, four series were investigated with $N=3,5,10$ and 15. The series with $N=3$ corresponds to the smallest scale of periodical heterogeneity because the first MHPM has a divergent flow inlet and a parallel flow outlet while the last MHPM has a parallel flow inlet and a convergent flow outlet. Only the in-between MHPM have the same parallel flow inlets and outlets (Figure 3). Therefore, $N=3,5,10$, and 15 correspond to $1,3,8$, and 13 periodical heterogeneities.

In this study, 15 MHPM columns were built, named A, B, C,..., N, O. For each series, four replicates of the step tracing experiment were conducted for four $90^{\circ}$ rotations of the study column, from which 
a mean breakthrough curve was deduced. The purpose of the $90^{\circ}$ rotation is to eliminate biases resulting from a possible interaction between density-driven effects and the $120^{\circ}$ spacing between the column inlets and outlets. The various column combinations used for the various experiments are summarized in Table 1.

\begin{tabular}{lllll}
\hline$N$ & $V_{0}(\mathrm{ml})$ & $L(\mathrm{~m})$ & Column groups & Total replicates \\
\hline 3 & 1383 & 0.45 & ABC, FGH, DEJ, KLM, INO & 20 \\
5 & 2305 & 0.75 & ABCDE, FGHIJ, KLMNO & 12 \\
10 & 4610 & 1.50 & A-J, F-O & 8 \\
15 & 6915 & 2.25 & A-O & 4 \\
\hline
\end{tabular}

Table 1. Experimental configurations. $L$ is the total length of the porous media.

\section{Experimental results}

\subsection{Experimental breakthrough curve}

For each value of $N$, four experimental signals are computed: the average breakthrough concentration $\bar{C}(t)$, its standard deviation $\sigma_{C}$, the minimum and maximum breakthrough concentrations, defined as

$$
\begin{array}{ll}
\bar{C}(t)=\frac{1}{R} \sum_{i=1}^{R} C_{i}(t), & \sigma_{C}^{2}=\frac{1}{R-1} \sum_{i=1}^{R}\left[C_{i}(t)-\bar{C}(t)\right]^{2}, \\
C_{\min }(t)=\min _{i} C_{i}(t), & C_{\max }(t)=\max _{i} C_{i}(t)
\end{array}
$$

where $C_{i}(t)$ is the concentration at time $t$ for the $i$ th replicate and $R$ is the number of replicates. The standard deviation and the difference between the minimum and maximum signals provide a measure of experiment variability.

Figure 4 (top) shows the experimental signals obtained for the four values $N=3,5,10$ and 15 . As expected from previously published studies (Danquigny et al., 2004; Huang et al., 1995; Saiers et al., 1994), the breakthrough curve for small $N$ strongly differs from the analytical S-shaped solution of the AD model (see the graphs for $N=3, N=5$ ). As $N$ increases, however, the breakthrough curves tend to behave similarly to a classical advection-dispersion model output ( $N=10$ and $N=15$ in Figure 4).

It is also worth stressing the strong variability of the breakthrough curve from one replicate to another for a given $N$. The amplitude of the standard deviation $\sigma_{C}(t)$ shown in Figure 4 is consistently about $5 \times 10^{-2}$ regardless of $N$. The amplitude of the min-max interval seems to decrease for larger $N$, but this might be due only to the smaller number of experiments (the min and max indicators being biased contrarily to the standard deviation).

This experimental result has two main consequences. First, it clearly shows that the observed behaviour (atypical or typical breakthrough curve) depends on the observation scale. Second, it leads to question the use of the $\mathrm{AD}$ approach to model solute transfer in porous media for small spatial scales. Indeed, the $\mathrm{AD}$ is adequate to reproduce breakthrough curves with a typical 'S' shape but not those with atypical behaviour. Several studies on heterogeneous porous media have already shown that the AD model is inadequate to describe the resulting breakthrough curves (Saiers et al., 1994; Huang et al., 1995; Danquigny et al., 2004; Li et al., 1994).

Additional information is brought by the Laplace transform of $\mathrm{d} C^{*} / \mathrm{d} t(t)$

$$
P(s)=s \int_{0}^{+\infty} C^{*}(t) \exp (-s t) \mathrm{d} t
$$

where $s$ is the Laplace variable. Since the input concentration is a unit step, $P(s)$ is the Laplace transform of the response of the $N$ columns to a concentration unit pulse. The graphs $P(s)$ are shown in Figure 4 (bottom). The graphs are drawn for $s \leq 0.1 \mathrm{~Hz}$ because the sampling time step is 5 seconds, thus making the numerical computation of (2) inaccurate for larger $s$. The response $P(s)$ behaves as a decreasing exponential for large $s$, as indicated by the linear asymptote in the graphs $(P$ is plotted using logarithmic coordinates). This is the typical signature of a delay. That the asymptotic linear 
behaviour is reached for smaller $s$ as $N$ increases indicates that advection phenomena become predominant as $N$ increases, a well-known feature of the advection-dispersion model for increasing Peclet number values. This, however, is not sufficient to validate the AD model, an issue that is addressed in Section 4.1.

\subsection{Scaling trend for the dispersion coefficient}

Questions (Q1) and (Q2) are answered assuming that the AD model is valid, with governing equations

$\frac{\partial C}{\partial t}+u \frac{\partial C}{\partial x}-D \frac{\partial^{2} C}{\partial x^{2}}=0$

$$
C(x, 0)=0 \quad \forall x \in[0, L]
$$

where $D$ is the dispersion coefficient, and $u$ the flow velocity. Analytical solutions are available for an upstream boundary condition in the form of a step flux function (Ogata and Banks 1961)

$$
C(0, t)=0 \forall t>0 \Rightarrow C(x, t)=\left[\operatorname{erfc}\left(\frac{x-u t}{2(D t)^{1 / 2}}\right)+\exp \left(\frac{u x}{D}\right) \operatorname{erfc}\left(\frac{x+u t}{2(D t)^{1 / 2}}\right)\right] \frac{C_{0}}{2}
$$

The unit response to a Dirac flux pulse is obtained as the derivative of (4) with respect to time

$$
C(0, t)=C_{0} \delta(t) \Rightarrow C(x, t)=\frac{C_{0}}{4 \pi^{1 / 2}(D t)^{3 / 2}}\left[(x+u t) \exp \left(-\frac{(x-u t)^{2}}{4 D t}\right)\right.
$$

$$
\left.+(x-u t) \exp \left(-\frac{(x+u t)^{2}}{4 D t}\right)\right]
$$

The Laplace transform of the solution (5) can also be obtained by solving equation (3) in the spaceLaplace domain:

$$
\hat{C}(x, s)=\frac{2}{1+F} \exp \left(\frac{u x}{D} \frac{1-F}{2}\right), F=\left(1+\frac{4 D}{u^{2} s}\right)^{1 / 2}
$$

The experimental dispersion coefficient $D$ is computed using a formula derived from the analytical solution of the advection-dispersion equation (Bear 1972):

$$
D=2\left(\frac{t_{60}-t_{40}}{t_{50}}\right)^{2} L u=2\left(\frac{t_{60}-t_{40}}{t_{50}}\right)^{2} \frac{L^{2}}{t_{50}}
$$

used by e.g. Irwin et al. (1996) and Sternberg et al. (1996).

Equation (5) was applied to the experimental configurations shown in Table 1. The detailed results are given in the Appendix (Tables A1 to A4), only statistics are presented here. For each value of $N$, the average, standard deviation, minimum and maximum of $D$ obtained from the various possible configurations are provided in Table 2. Several remarks can be made at this stage.

Firstly, the variations in the average $D$ with $L$ are smaller than the standard deviation of $D$ for any given $L$. Since the individual variations between the replicates for a given $N$ override by far any visible trend, it can be concluded that no significant trend of distance-dependent dispersion $D(L)$ is detected given the experimental uncertainty.

Secondly, given the range of variations between the minimum and maximum values, it is possible to find a sequence among all the experimental values in Tables A1-4 such that an increasing trend $D(L)$ is detected. An example of such a sequence is (ABC1, ABCDE2, A-J4, A-O4). But it would be just as easy to detect a decreasing trend $D(L)$ by choosing the values in Tables A1-4 appropriately, for instance the sequence (KLM4, FGHIJ3, K-O2, A-O3). Such trends, however, would only be sampling artefacts.

It can be concluded as an answer to question (Q1) that, in the limit of experimental uncertainty, no significant scaling trend can be identified for the dispersion coefficient in the present MHPM. As an 


\begin{tabular}{cccccc}
\hline$L(\mathrm{~m})$ & $N$ & $D\left(\mathrm{~m}^{2} \mathrm{~s}^{-1}\right)$ average & $\begin{array}{c}D\left(\mathrm{~m}^{2} \mathrm{~s}^{-1}\right) \text { standard } \\
\text { deviation }\end{array}$ & $D\left(\mathrm{~m}^{2} \mathrm{~s}^{-1}\right)$ minimum & $D\left(\mathrm{~m}^{2} \mathrm{~s}^{-1}\right)$ maximum \\
\hline 0.45 & 3 & $1.08 \times 10^{-4}$ & $3.65 \times 10^{-5}$ & $5.60 \times 10^{-5}$ & $1.69 \times 10^{-4}$ \\
0.75 & 5 & $1.36 \times 10^{-4}$ & $6.37 \times 10^{-5}$ & $7.40 \times 10^{-5}$ & $3.29 \times 10^{-4}$ \\
1.50 & 10 & $1.18 \times 10^{-4}$ & $3.38 \times 10^{-5}$ & $6.58 \times 10^{-5}$ & $1.81 \times 10^{-4}$ \\
2.25 & 15 & $1.18 \times 10^{-4}$ & $3.00 \times 10^{-5}$ & $8.43 \times 10^{-5}$ & $1.67 \times 10^{-4}$ \\
\hline
\end{tabular}

answer to question (Q2), sampling artefacts may easily lead to the conclusion that the dispersion coefficient is subjected to a scaling trend in this MHPM. It is stressed that only the systematic replication of the tracer experiments and a high-resolution sampling allows this question to be answered with a satisfactory degree of confidence.

Table 2. AD model. Dispersion coefficients obtained using equation (7). See the detailed results in the Appendix.

\subsection{Unit transfer function}

The purpose of the present subsection is to answer question (Q3). Since the various replicates $C_{i}(t)$ all give different time series for a given number $N$ of columns, the unit transfer function is different for each replicate. The proposed approach consists in determining the envelope of all possible $C(t)$ signals for a Dirac input. It is obvious from Definition (1) that neither the lower bound $C_{\min }$ nor the upper bound $C_{\max }$ of this envelope do correspond to a single replicate signal $C_{i}(t)$, the purpose being only to determine the range of possible variations of $C(t)$ for each time $t$.

For this, it is necessary to estimate the unit transfer function of a single MHPM period. Denote by $P_{N, i}$ the Laplace transform of the $i$ th replicate for a number $N$ of columns. A minimum-maximum confidence interval is defined for $P$ with the following lower and upper bounds:

$$
P_{N, \min }=\min _{i=1, \ldots, R} P_{N, i}, \quad P_{N, \max }=\max _{i=1, \ldots, R} P_{N, i}
$$

where $N$ denotes the number of columns used in the experiment and $i$ denotes the replicate. This allows a confidence interval to be defined for the unit Laplace transform of a single MPMH period. Let $r$ be the Laplace transform of a single MHPM period for a Dirac concentration input. The lower and upper bounds defined in (8) yield a confidence interval $\left[r_{\min }(M, N), r_{\max }(M, N)\right]$ for $r$. Indeed, the convolution property of the Laplace transform leads to

$$
\begin{aligned}
& r_{\text {min }}(M, N) \leq r \leq r_{\text {max }}(M, N) \quad \forall(M, N), M<N \\
& r_{\text {min }}(M, N)=\left(\frac{P_{N, \text { min }}}{P_{M, \text { max }}}\right)^{\frac{1}{N-M}}, r_{\text {max }}(M, N)=\left(\frac{P_{N, \text { max }}}{P_{M, \text { min }}}\right)^{\frac{1}{N-M}}, M<N
\end{aligned}
$$

If a unit transfer function with scale-independent parameters exists, all the confidence intervals $\left[r_{\min }(M, N), r_{\max }(M, N)\right]$ derived for all possible combinations $(M, N)$ should overlap. Denoting respectively by $r_{1}$ and $r_{2}$ the lower and upper bounds of the intersection of all the confidence intervals, one has by definition

$$
r_{1}=\max _{M, N} r_{\min }(M, N), \quad r_{2}=\min _{M, N} r_{\max }(M, N)
$$

The confidence intervals overlap provided that $r_{1}(s)<r_{2}(s) \forall s$.

Figures $5 a-f$ show the min-max confidence intervals derived from equation (9b) for all the possible $(M, N)$ combinations. It is noted that the confidence interval becomes narrower as the number $(N-M)$ of periods used to determine the unit transfer function increases, thus indicating the convergence of the estimates (9b).

The intersection of all the confidence intervals is shown in Figure 5g. The non-zero width of the interval $\left[r_{1}, r_{2}\right]$ indicates that a transfer function with scale-independent parameters can indeed be found within the range $N=3$ to $N=15$. In other words, it is possible to find a scalar transport model with spatially uniform parameters that yields simulation results lying within the experimental 
uncertainty. This model, however, is not necessarily the classical AD model. This issue is explored in the next section.

\section{Discussion}

\subsection{Validity of the AD model}

The purpose of the present section is to answer questions (Q4-5). If the AD model is valid at the scale of $N$ MHPM periods, then there exists a combination $(D, u)$ such that

$$
r_{1}^{N}(s) \leq \hat{C}(N l, s) \leq r_{2}^{N}(s) \forall s
$$

where $l=15 \mathrm{~cm}$ is the length of a single MHPM period. Clearly, this condition is not satisfied for $N=1$. Indeed, for large $s$, the analytical solution (6) behaves as

$$
F \approx \frac{2}{u} D^{1 / 2} s^{1 / 2} \Rightarrow \hat{C}(N l, s) \approx \frac{u}{D^{1 / 2} s^{1 / 2}} \exp \left(-\frac{N l}{D^{1 / 2}} s^{1 / 2}\right)
$$

a function with a horizontal asymptotic direction in semi-logarithmic coordinates, while $r_{1}(s)$ and $r_{2}(s)$ take the form of straight lines for large $s$ (see Figure $5 \mathrm{~g}$ ). Consequently, the function $\hat{C}(N l, s)$ does not lie within the interval $\left[r_{1}^{N}, r_{2}^{N}\right]$ for all $s$ and the AD model is invalid to some extent. This is illustrated by Figure 6a, where the confidence interval $\left[r_{1}^{N}, r_{2}^{N}\right]$ and the function $\hat{C}(N l, s)$ are plotted for $N=1$.

The degree of accuracy of the AD model is assessed by computing the L2-norm of the difference between the experimental transfer function and the theoretical one in the Laplace space:

$$
\begin{aligned}
& L_{2}=\left[\frac{1}{S} \int_{0}^{S} \varepsilon^{2}(s) \mathrm{d} s\right]^{1 / 2} \\
& \varepsilon(s)=\left\{\begin{array}{ccc}
\hat{C}(N l, s)-r_{1}^{N}(s) & \text { if } & \hat{C}(N l, s)<r_{1}^{N}(s) \\
0 & \text { if } & r_{1}^{N}(s) \leq \hat{C}(N l, s) \leq r_{2}^{N}(s) \\
\hat{C}(N l, s)-r_{2}^{N}(s) & \text { if } & \hat{C}(N l, s)>r_{2}^{N}(s)
\end{array}\right.
\end{aligned}
$$

where $S=0.1 \mathrm{~Hz}$ is the upper bound of $s$. Eq. (13b) gives a zero error if the solution of the AD model lies between $r_{1}$ and $r_{2}$ in the Laplace space, that is, if the $\mathrm{AD}$ solution falls within the range of the experimental uncertainty.

Table 3 shows the combinations $(D, u)$ that minimize the $L_{2}$-norm (13a) for various values of $N$. Figure 6a-g shows the corresponding plots in the Laplace space. The $L_{2}$-norm for $N=1$ is large, indicating that the AD model provides a poor description of the transfer process at the scale of a single column. However, the $L_{2}$-norm decreases quickly with $N$, which is an indication that the $\mathrm{AD}$ model gains worth with the number of MHPM periods. The driving mechanism for this is interesting to notice. As shown by Figures 6a-g, the AD transfer function lies outside the confidence interval for $s>3 \times 10^{-2} \mathrm{~Hz}$ in all graphs. In other words, in terms of relative error, the AD model is equally inaccurate for all values of $N$. However, the amplitude of the transfer function decreases quickly as $N$ increases. The absolute value of the error thus becomes smaller as $N$ increases.

\begin{tabular}{ccccc}
\hline$N$ & $D$ & $u$ & $L_{2}$ & Figure \\
\hline 1 & $1.57 \times 10^{-4}$ & $1.98 \times 10^{-3}$ & $2.13 \times 10^{-3}$ & $6 \mathrm{a}$ \\
3 & $2.85 \times 10^{-4}$ & $1.18 \times 10^{-3}$ & $1.27 \times 10^{-4}$ & $6 \mathrm{~b}$ \\
5 & $2.36 \times 10^{-4}$ & $9.98 \times 10^{-4}$ & $1.70 \times 10^{-6}$ & $6 \mathrm{c}$ \\
10 & $2.24 \times 10^{-4}$ & $7.97 \times 10^{-4}$ & $1.75 \times 10^{-10}$ & $6 \mathrm{~d}$ \\
15 & $2.07 \times 10^{-4}$ & $7.71 \times 10^{-4}$ & $6.51 \times 10^{-15}$ & $6 \mathrm{e}$ \\
20 & $2.07 \times 10^{-4}$ & $7.71 \times 10^{-4}$ & $2.045 \times 10^{-18}$ & $6 \mathrm{f}$ \\
30 & $2.07 \times 10^{-4}$ & $7.71 \times 10^{-4}$ & $2.66 \times 10^{-25}$ & $6 \mathrm{~g}$ \\
\hline
\end{tabular}

Table 3. AD model. Optimal parameter set for the objective function (13a-b). 
Note that the value for $D$ is found to be consistently about $2 \times 10^{-4} \mathrm{~m}^{2} \mathrm{~s}^{-1}$ (except for $N=3$ ), while formula (7) gives an average value of about $1.2 \times 10^{-4} \mathrm{~m}^{2} \mathrm{~s}^{-1}$ (see Table 2). This striking difference between the two sets of values can be explained by the calibration approach. The values given in Table 1 are computed separately for each individual experiment, under the assumption that the AD model provides an accurate description of the transport processes for each experiment. However, a $(D, u)$ combination calibrated for a given replicate will fail totally to predict the behaviour of another replicate.

In contrast, the dispersion coefficient values in Table 3 account for the variability of experimental uncertainty. It is not requested that the $\mathrm{AD}$ model should satisfy each individual experiment as accurately as possible, but that its response should lie within the intersection of all the confidence intervals associated with different MHPM lengths. As a consequence, smoother unit responses (thus larger dispersion coefficient values) are allowed compared to the former approach. Moreoever, both calibration approaches use different variables (pointwise $C(t)$ values in the first approach, $\hat{C}(N l, s)$ over an interval in the second approach).

\subsection{Validity of alternate transport models}

This section is motivated by Question (Q6). Since the classical AD model fails to reproduce the unit pulse response of the column, the question arises whether alternate transport models may be more successful. To start with, examining Figure (5g) indicates an asymptotically linear behaviour in semilogarithmic coordinates. In other words, the unit transfer function is asymptotically equivalent to a decreasing exponential in the Laplace space. The experimental unit transfer function thus verifies the following property:

$$
\hat{C}(x, s) \underset{s \rightarrow \infty}{\approx} A \exp (-B x s) \Rightarrow C(x, t) \underset{t \rightarrow 0}{\approx} H(t-B x) f(t)
$$

where $A$ and $B$ are constants and $H$ is Heaviside's step function. In other words, the signal propagates at a finite velocity $B^{-1}$, with a breakthrough concentration rising from 0 at $t=B x$ to 1 for $t \rightarrow \infty$. The inadequacy of the $\mathrm{AD}$ model for small times and distances precisely stems from the infinite front propagation speed associated with the Fickian flux model.

The Mobile-Immobile (MI) model (Van Genuchten and Wierenga, 1977) is one of the earliest and most probably best-known non-Fickian models for dispersion. Later on, generalizations such as the multirate model (Haggeerty and Gorelick, 1995) have been proposed. The governing equations for the MI model are:

$$
\begin{aligned}
& \frac{\partial C_{m}}{\partial t}+u \frac{\partial C_{m}}{\partial x}-D \frac{\partial^{2} C}{\partial x^{2}}=\frac{k}{\theta}\left(C_{i}-C_{m}\right) \\
& \frac{\partial C_{i}}{\partial t}=\frac{k}{1-\theta}\left(C_{m}-C_{i}\right)
\end{aligned}
$$

where $C_{i}$ and $C_{m}$ are respectively the concentrations in the immobile and mobile regions, $k$ is an exchange rate constant and $\theta$ is the relative volume fraction of the mobile region. Applying the Laplace transform in time, eliminating $C_{i}$ yields

$$
\begin{aligned}
& \frac{\mathrm{d}^{2} \hat{C}_{m}}{\mathrm{~d} x^{2}}-\frac{u}{D} \frac{\mathrm{d} \hat{C}_{m}}{\mathrm{~d} x}-a \frac{s}{D} \hat{C}_{m}=0, \quad a=\frac{k+(1-\theta) \theta s}{[k+(1-\theta) s] \theta} \\
& \hat{C}_{m}(x, s)=\hat{C}_{m}(0, s) \exp (-\lambda x), \quad \lambda=\left[\left(\frac{u}{2 D}\right)^{2}+\frac{a s}{D}\right]^{1 / 2}-\frac{u}{2 D}
\end{aligned}
$$

For large $s$, the following equivalence is obtained:

$$
\underset{s \rightarrow \infty}{\approx} 1 \Rightarrow \tilde{C}_{m}(x, s) \underset{s \rightarrow \infty}{\approx} A \exp \left(-\frac{s^{1 / 2}}{D^{1 / 2}} x\right)
$$


leading to a horizontal asymptotic direction in the semi-logarithmic plots of Figures 4 to 6 .

2 Consequently, the $\hat{C}_{m}(x, s)$ curve (16) cannot remain between the min-max bounds of the experimental confidence interval for large $s$, thereby failing to account for the experimental MHPM behaviour for small times.

The modelling community's attention has been drawn over the past years to anomalous transport models (see e.g. Gao et al, 2009 for a review). Anomalous transport models use a non-Fickian model for dispersion. The underlying assumption is that the characteristic time scale and/or the characteristic length of the solute movement are infinite. Under such conditions, the assumption of Brownian movement is no longer valid and long-term memory effects appear. The general form for anomalous transport models is

$$
\frac{\partial C}{\partial t}+u \frac{\partial^{\beta}}{\partial t^{\beta}} \frac{\partial C}{\partial x}-D \frac{\partial^{1-\alpha}}{\partial t^{1-\alpha}} \frac{\partial^{\gamma} C}{\partial x^{\gamma}}=0, \quad\left\{\begin{array}{c}
0<\alpha \leq 1 \\
0<\gamma \leq 1 \\
\beta \in\{1-\alpha, 1\}
\end{array}\right.
$$

where $\partial^{k} / \partial t^{k}$ stands for the $k$ th-order fractional time derivative (see e.g. Oldham and Spanier, 1974; Podlubny, 1999 for definitions). For $\alpha<1, \gamma<1$, Equation (18) is obtained assuming that the probability density function $w$ of the displacement time and $d$ of displacement length obey the following asymptotic behaviours for large time and distances (Metzler and Klafter, 2000):

$$
w(t) \underset{t \rightarrow+\infty}{\approx} \frac{A}{t^{1+\alpha}}, \quad d(x) \underset{x \rightarrow+\infty}{\approx} \frac{B}{x^{1+\gamma}}
$$

The combinations $(\beta=1-\alpha, \gamma=1)$ and $(\beta=1, \gamma=1)$ correspond respectively to the so-called Galileanvariant and Galilean-invariant fractional diffusion models presented in Metzler et al. (2002) under the Continuous Time Random Walk (CTRW) formalism. For $\gamma<1$, so-called Levy walks are obtained. As shown in Klafter et al. (1987), that solute particles may cover arbitrary long distances within a displacement (thus leading to infinite velocities, a physical impossibility) may be balanced by a coupling between the pdfs $w(t)$ and $d(x)$. Depending on the respective natures of $w$ and $d$, any anomalous dispersive behaviour may be obtained, from subdiffusive to superdiffusive. Note that the case $(\alpha=\beta=1, \gamma<1)$ has been investigated by Pachepsky et al. (2000) and applied to column experiment simulation by Gao et al. (2009). The behaviour of the analytical expression given by Pachepsky et al. (2009) is not easy to analyze, even in the Laplace space. However, a qualitative reasoning suffices to discard this option to model the present experiments. The Levy flight model yields infinite signal propagation velocities, just as does the standard, Fickian model. Consequently, for large $s$, this model also lies outside the experimental unit response of the MHPM in the Laplace space. The case $\gamma$ will therefore not be analyzed hereafter.

For $\gamma=1$, applying the Laplace transform to (18) gives

$$
\frac{\mathrm{d}^{2} \hat{C}}{\mathrm{~d} x^{12}}-\frac{u}{D} s^{1-\alpha-\beta} \frac{\mathrm{d}^{\beta} \hat{C}}{\mathrm{~d} x^{\beta}}-\frac{s^{\alpha}}{D} \hat{C}=0
$$

$$
\hat{C}(x, s)=\hat{C}(0, s) \exp (-\lambda x), \quad \lambda=\left[\left(\frac{u}{2 D}\right)^{2} s^{2-2 \alpha-2 \beta}+\frac{s^{\alpha}}{D}\right]^{1 / 2}-\frac{u}{2 D} s^{1-\alpha-\beta}
$$

which gives the following equivalences

$$
\beta \in\{1-\alpha, 1\} \Rightarrow \lambda \underset{s \rightarrow+\infty}{\approx} \frac{s^{\alpha / 2}}{D^{1 / 2}} \Rightarrow \tilde{C}(x, s) \underset{s \rightarrow+\infty}{\approx} A \exp \left(-\frac{s^{\alpha / 2}}{D^{1 / 2}} x\right)
$$

As for the MI model, this model yields a horizontal asymptotic direction in semi-logarithmic coordinates and does not allow the experimental behaviour to be reproduced for large $s$. 
Although attractive in theory, anomalous transport models may fail in a number of cases, not because they are not valid in general, but because they are not applied at the right space/time scales. Fractional derivatives are introduced in anomalous transport models under the assumption of heavy-tailed waiting time and travel distances probability density functions. The CTRW analysis (Klafter et al., 1987; Metzler and Klafter, 2000) shows that fractional derivatives stem naturally from this assumption as the Laplace variable $s$ tends to zero. For the CTRW analysis to be valid, the observation time and/or travelled distance must be sufficient for a significantly large number of solute molecule jumps to occur. Moreover, the solute must cover a sufficient number of medium heterogeneities so that the ergodicity of the transport process be ensured. This underlying assumption is in contradiction with the present experimental setup, that obviously deals with space and time scales smaller than those required for ergodicity. As mentioned by Gao et al. (2009), the reason why anomalous transport models may succeed better to reproduce experimental column behaviour may simply be that they incorporate more degrees of freedom than classical transport models. As stressed by these authors, more degrees of freedom do not necessary induce a better model.

\section{Conclusions}

This paper presents the results of intermediate scale tracer experiments carried out using a model heterogeneous porous medium with a variable length. The periodic character of the medium allows the stationarity of the medium properties to be preserved. The large number of replicates (44 experiments for 4 different column lengths) allows sampling artefacts to be minimized and reliable statistics to be derived. A confidence interval for the unit transfer function of a single period of the model medium is derived from a Laplace transform analysis.

In the light of this work, the answers to questions (Q1-6) raised in the Introduction are the following.

(A1) No clear scaling trend can be identified for the dispersion coefficient within the range of experimental uncertainty for the present MHPM.

(A2) Given the strong individual variability observed from one replicate to another, sampling artefacts may lead to wrongly conclude that the dispersion coefficient is scale-dependent.

(A3) There exists a model with scale-independent parameters that can provide a correct description of the solute transfer in the MHPM from $N=3$ to $N=15$.

(A4) This model is not the AD model, because it does not allow the experimental breakthrough curves to be reproduced accurately within the range of the experimental uncertainty.

(A5) The AD model can be deemed to provide an accurate description of the present MHPM after the solute has been transported over at least 10 (and most probably 20) periods. The size of the REV for this MHPM is thus between 10 and 20 MHPM periods.

(A6) The MI and CTRW models will fail to reproduce the small-time and short-distance experimental behaviour of the MHPM because of the second-order derivative used in the modelling of dispersion.

We should like to stress the strong variability of the experimental results, even in the case of the present artificial MHPM, that was intentionally made strictly periodic. This should make modellers extremely cautious when dealing with natural media, the geometry of which is often poorly known and does not exhibit the degree of determinism of the present MHPM. This makes the systematic replication of breakthrough experiments indispensable in seeking reliable conclusions on scaling effects, as well as in model calibration attempts. It is a useful reality check of the assumptions of stationarity and ergodicity (Kulasiri and Verwoerd, 2002) of the AD model. From a more general perspective, one might also question the relevance of investigating the scaling behaviour of the AD model for spatial scales where it is invalid. This questioning also holds for alternate models such as anomalous transport models.

Lastly, the present work shows the usefulness of a high resolution sampling in analyzing the MHPM transfer functions (each of the breakthrough curves used in this study is made of 500 to 1500 records).

Further research focuses on (i) an experimental design from which the boundary effects arising from the inlet distribution is absent, which would allow the unit response $r$ to be derived directly for a single 
1 column, (ii) carrying out series of experiments at identical $N$ for several discharge values, so as to analyze the dependence of the dispersion coefficient on the flow velocity, and (iii) developing and applying alternative transport models (among which variations of the multirate model) to better simulate the ISE using scale-independent parameters. The present experiments clearly indicate that such a model exists. However, it should most probably not be based on a Fickian description of dispersion.

\section{Acknowledgements}

8 The authors would like to thank Dr Philippe Ackerer, Research Director at LHYGEES Strasbourg,

9 France, and Jonas Darey for assisting in the experimental work.

\section{Appendix. Dispersion coefficient - detailed results}

\begin{tabular}{ccccc}
\hline Configuration & $t_{40}(\mathrm{~s})$ & $t_{50}(\mathrm{~s})$ & $t_{60}(\mathrm{~s})$ & $D\left(\mathrm{~m}^{2} \mathrm{~s}^{-1}\right)$ \\
\hline ABC1 & 495 & 585 & 680 & $6.92 \times 10^{-5}$ \\
ABC2 & 485 & 575 & 660 & $6.52 \times 10^{-5}$ \\
ABC3 & 485 & 580 & 660 & $6.36 \times 10^{-5}$ \\
ABC4 & 430 & 545 & 675 & $1.50 \times 10^{-4}$ \\
DEJ1 & 395 & 500 & 615 & $1.57 \times 10^{-4}$ \\
DEJ2 & 450 & 550 & 635 & $8.33 \times 10^{-5}$ \\
DEJ3 & 415 & 515 & 610 & $1.13 \times 10^{-4}$ \\
DEJ4 & 470 & 560 & 645 & $7.06 \times 10^{-5}$ \\
FGH1 & 515 & 605 & 690 & $5.60 \times 10^{-5}$ \\
FGH2 & 465 & 560 & 665 & $9.22 \times 10^{-5}$ \\
FGH3 & 480 & 600 & 725 & $1.12 \times 10^{-4}$ \\
FGH4 & 460 & 595 & 735 & $1.45 \times 10^{-4}$ \\
IHO1 & 425 & 555 & 665 & $1.36 \times 10^{-4}$ \\
IHO2 & 445 & 530 & 615 & $7.86 \times 10^{-5}$ \\
IHO3 & 400 & 510 & 620 & $1.48 \times 10^{-4}$ \\
IHO4 & 455 & 555 & 645 & $8.55 \times 10^{-5}$ \\
KLM1 & 435 & 545 & 675 & $1.44 \times 10^{-4}$ \\
KLM2 & 480 & 575 & 680 & $8.52 \times 10^{-5}$ \\
KLM3 & 435 & 550 & 680 & $1.46 \times 10^{-4}$ \\
KLM4 & 420 & 545 & 680 & $1.69 \times 10^{-4}$ \\
\hline
\end{tabular}

11 Table A1. AD model. Dispersion coefficients obtained using equation (5) for $N=3$. 


\begin{tabular}{ccccc}
\hline Configuration & $t_{40}(\mathrm{~s})$ & $t_{50}(\mathrm{~s})$ & $t_{60}(\mathrm{~s})$ & $D\left(\mathrm{~m}^{2} \mathrm{~s}^{-1}\right)$ \\
\hline ABCDE1 & 865 & 1005 & 1165 & $9.97 \times 10^{-5}$ \\
ABCDE2 & 900 & 1035 & 1170 & $7.40 \times 10^{-5}$ \\
ABCDE3 & 725 & 860 & 1010 & $1.44 \times 10^{-4}$ \\
ABCDE4 & 730 & 870 & 1020 & $1.44 \times 10^{-4}$ \\
FGHIJ1 & 890 & 1035 & 1185 & $8.83 \times 10^{-5}$ \\
FGHIJ2 & 840 & 970 & 1100 & $8.33 \times 10^{-5}$ \\
FGHIJ3 & 845 & 1035 & 1225 & $1.46 \times 10^{-4}$ \\
FGHIJ4 & 765 & 905 & 1065 & $1.37 \times 10^{-4}$ \\
KLMNO1 & 765 & 910 & 1065 & $1.34 \times 10^{-4}$ \\
KLMNO2 & 835 & 985 & 1140 & $1.10 \times 10^{-4}$ \\
KLMNO3 & 805 & 980 & 1165 & $1.55 \times 10^{-4}$ \\
KLMNO4 & 630 & 825 & 1035 & $3.29 \times 10^{-4}$ \\
\hline
\end{tabular}

4

\begin{tabular}{ccccc}
\hline Configuration & $t_{40}(\mathrm{~s})$ & $t_{50}(\mathrm{~s})$ & $t_{60}(\mathrm{~s})$ & $D\left(\mathrm{~m}^{2} \mathrm{~s}^{-1}\right)$ \\
\hline A-J1 & 1730 & 1965 & 2215 & $1.40 \times 10^{-4}$ \\
A-J2 & 1850 & 2090 & 2320 & $1.09 \times 10^{-4}$ \\
A-J3 & 1695 & 1960 & 2245 & $1.81 \times 10^{-4}$ \\
A-J4 & 1800 & 2035 & 2280 & $1.23 \times 10^{-4}$ \\
K-O1 & 1925 & 2125 & 2350 & $8.47 \times 10^{-5}$ \\
K-O2 & 1805 & 2015 & 2235 & $1.02 \times 10^{-4}$ \\
K-O3 & 1870 & 2050 & 2225 & $6.58 \times 10^{-5}$ \\
K-O4 & 1675 & 1905 & 2140 & $1.41 \times 10^{-4}$ \\
\hline
\end{tabular}

5 6

\begin{tabular}{ccccc}
\hline Configuration & $t_{40}(\mathrm{~s})$ & $t_{50}(\mathrm{~s})$ & $t_{60}(\mathrm{~s})$ & $D\left(\mathrm{~m}^{2} \mathrm{~s}^{-1}\right)$ \\
\hline A-O1 & 2800 & 3075 & 3360 & $1.09 \times 10^{-4}$ \\
A-O2 & 2655 & 2925 & 3185 & $1.14 \times 10^{-4}$ \\
A-O3 & 2785 & 3025 & 3265 & $8.43 \times 10^{-5}$ \\
A-O4 & 2680 & 3010 & 3350 & $1.67 \times 10^{-4}$ \\
\hline
\end{tabular}

\section{References}

Table A2. AD model. Dispersion coefficients obtained using equation (5) for $N=5$.

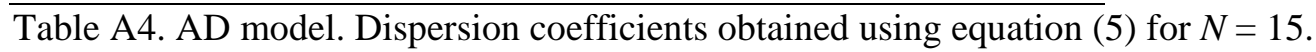

Bear, J., 1972. Dynamics of fluids in porous media. Dover, NY.

Dagan, G., 1989. Flow and Transport in Porous Formations, Springer-Verlag, Berlin. reconstructed heterogeneous porous media. Journal of Hydrology, 294, 196-212. 377, 391-404.

Danquigny, C., Ackerer, P., and Carlier, J. P., 2004. Laboratory tracer tests on three-dimensional

Gao, G., Zhan, H., Feng, S., Huang, G., Mao, X., 2009. Comparison of alternative models for simulating anomalous solute transport in a large heterogeneous soil column. Journal of Hydrology, 
Gao, G., Zhan, H., Feng, S., Fu, B., Ma, Y., Huang, G., 2010. A new mobile-immobile model for reactive solute transport with scale-dependent dispersion. Water Resources Research, 46, W08533.

Gelhar, L. W., W. Welty, Rehfeldt, K.R., 1992. A critical review of data on field-scale dispersion in aquifers, Water Resources Research, 28, 1955-1974.

Golfier, F., Quintard, M., and Woodd, B. D., 2011. Comparison of theory and experiment for solute transport in weakly heterogeneous bimodal porous media. Advances in Water Resources, 34, 899-914.

Greiner, A., Schreiber, W., Brix, G., and Kinzelbach, W., 1997. Magnetic resonance imaging of paramagnetic tracers in porous media: Quantification of flow and transport parameters. Water Resources Research, 33, 6, 1461-1473.

Haggerty, R., Gorelick, S. M. 1995. Multiple - rate mass transfer for modeling diffusion and surface reactions in media with pore - scale heterogeneity, Water Resour. Res., 31, 2383-2400.

Huang, K., Toride, N., Van Genuchten, M. T., 1995. Experimental investigation of solute transport in large, homogeneous and heterogeneous, saturated soil columns. Transport in Porous Media, 18, 283302.

Irwin, N. C., Botz, M. M., and Greenkorn, R. A., 1996. Experimental investigation of characteristic length scale in periodic heterogeneous porous media. Transport in Porous Media, 25, 235-246.

Klafter, J., Blumen, A., Shlesinger, M.F, 1987. Stochastic pathway to anomalous difusion. Physical Review A, 35, 3081-3085.

Kulasiri, D., Verwoerd, W., 2002. Stochastic dynamics - modelling solute transport in porous media. North Holland Series Applied Mathematics volume 44, Elsevier.

Li, L., Barry, D. A., Culligan-Hensley, P. J., and Bajracharya, K., 1994. Mass transfer in soils with local stratification of hydraulic conductivity. Water Resources Research, 30, 11, 2891-2900.

Metzler, R., Klafter, J., 2000. The random walk's guide to anomalous diffusion: a fractional dynamics approach. Physics Reports, 339, 1-77.

Niehren, S., Kinzelbach, W., 1998. Artificial colloid tracer tests: development of a compact on-line microsphere counter and application to soil column experiments. Journal of Contaminant Hydrology, 35, 249-259.

Ogata, A., Banks, R.B., 1961. A solution of the differential equation of longitudinal dispersion in porous media. US Geological Survey Professional Paper 411A.

Oldham, K.B., Spanier, J., 1974. The fractional Calculus. Academic Press.

Pachepsky, Y., Benon, D., Rawls, W., 2000. Simulating scale-dependent solute transport in soils with the fractional advective-dispersive equation. Soil Science Society of America Journal, 64, 1234-1243.

Podlublny, I., 1999. Fractional Differential Equations. An introduction to fractional derivatives. Academic Press.

Saiers, J.E., Hornberger, G.M., Hervey, C., 1994. Colloidal silica transport through structured, heterogeneous porous media. Journal of Hydrology, 163, 271-288.

Silliman, S.E., Simpson, E.S., 1987. Laboratory evidence of the scale effect in dispersion of solutes in porous media. Water Resources Research, 23(8), 1667-1673. 
Silliman, S.E., Zheng, L., Conwell, P., 1998. The use of laboratory experiments for the study of conservative solute transport in heterogeneous porous media. Hydrogeology Journal, 6, 166-177.

Sternberg, S. P. K., Cushman, J., and Greenkorn, R. A., 1996. Laboratory observation of nonlocal dispersion. Transport in Porous Media, 23, 135-151.

Tran Ngoc, T. D., Lewandowska, J., Vauclin, M., and Bertin, H., 2011. Two-scale modeling of solute dispersion in unsaturated double-porosity media: Homogenization and experimental validation. International Journal for Numerical and Analytical Methods in Geomechanics, 35, 1536-1559.

Van Genuchten, M. T., Wierenga, P.G., 1977. Mass transfer studies in sorbing porous media: II. Experimental evaluation with tritium (3H2O), Soil Sci. Soc. Am. J., 41, 272-278.

Zhou, L., Selim, H.M., 2003. Scale-dependent dispersion in soils: an overview, Adv. Agron., 80, 223263.

Zin, B., Meigs, L. C., Harvey, C. F., Haggerty, R., Peplinsky, W. J., Von Schwerin, C. F., 2004. Experimental visualization of solute transport and mass transfer processes in two-dimensional conductivity fields with connected regions of high conductivity. Environ. Sci. Technol., 38, 39163926. 


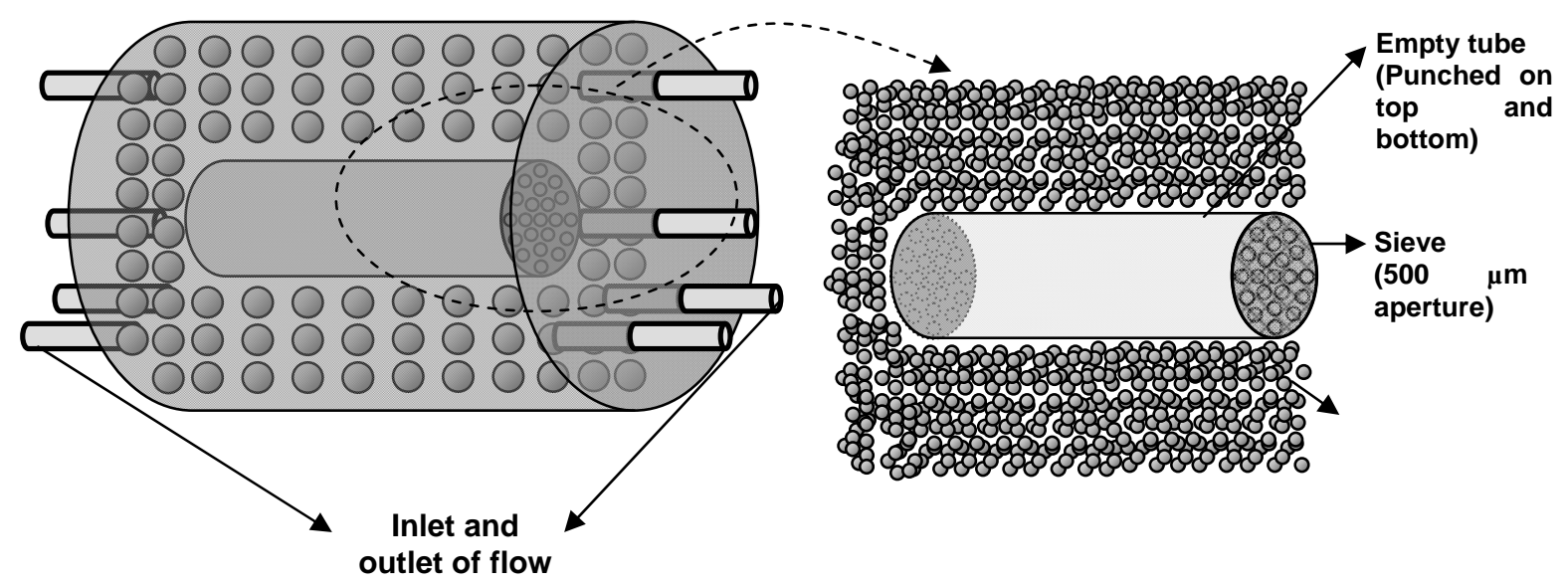

(a)

(b)

Figure 1. (a) A model heterogeneous porous media (MHPM) with 4 inlets and outlets that occupy the centre of gravity and the 3 vertices of an equilateral triangle. The centre of gravity is on the central axis of the column and the 3 vertices are $1 \mathrm{~cm}$ away from the column periphery. (b) The internal cavity is a tube that is punched on top and bottom and covered with a $500 \mu \mathrm{m}$ sieve. The sieve impedes the glass spheres from entering inside the cavity and guaranties that only water (and solute) can transfer through the cavity. 

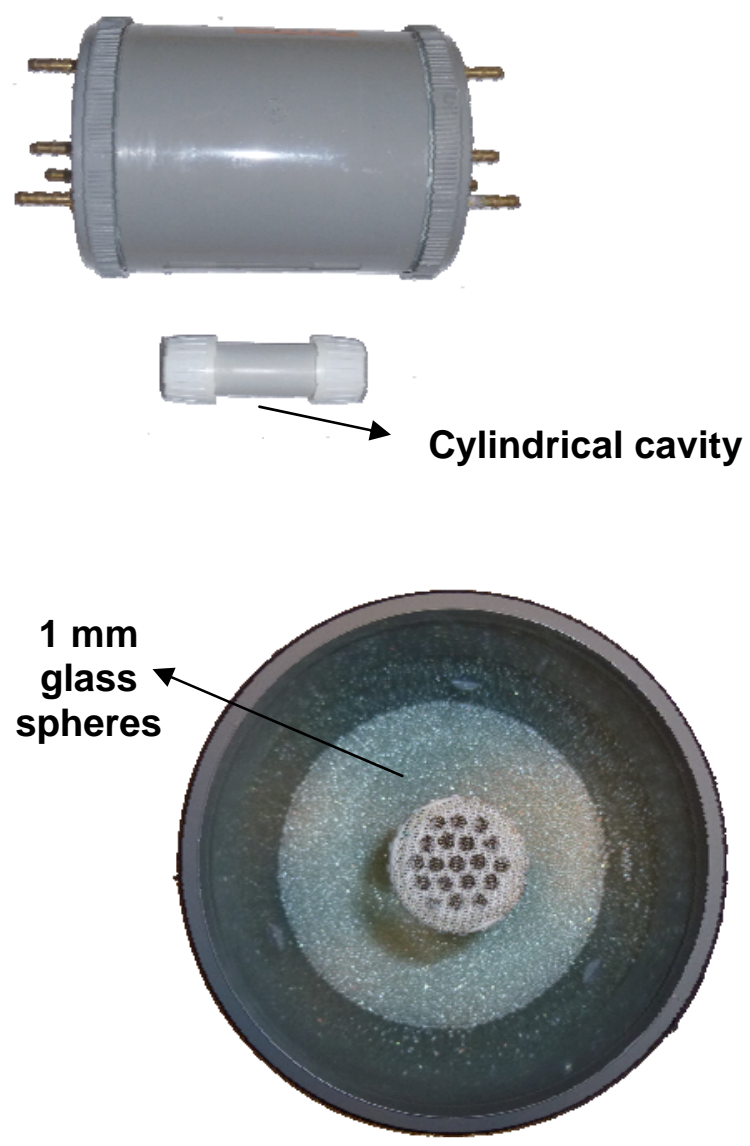

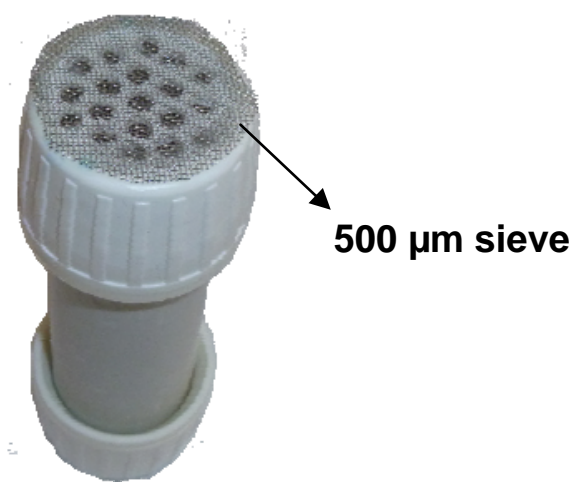

4 inlets/outlets

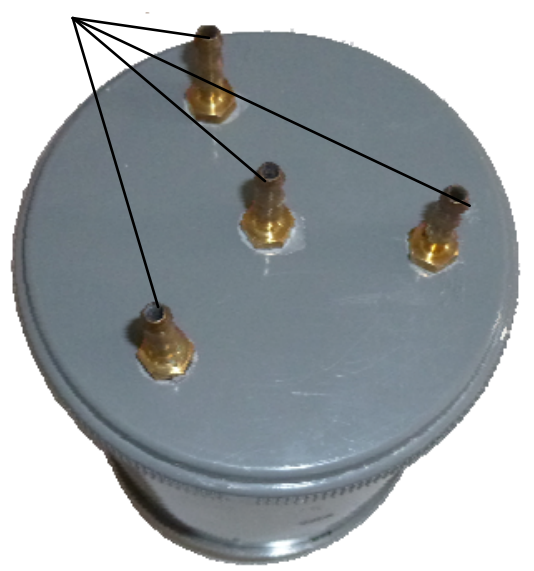

3

4

5

Figure 2. Real captures of a MHPM. 


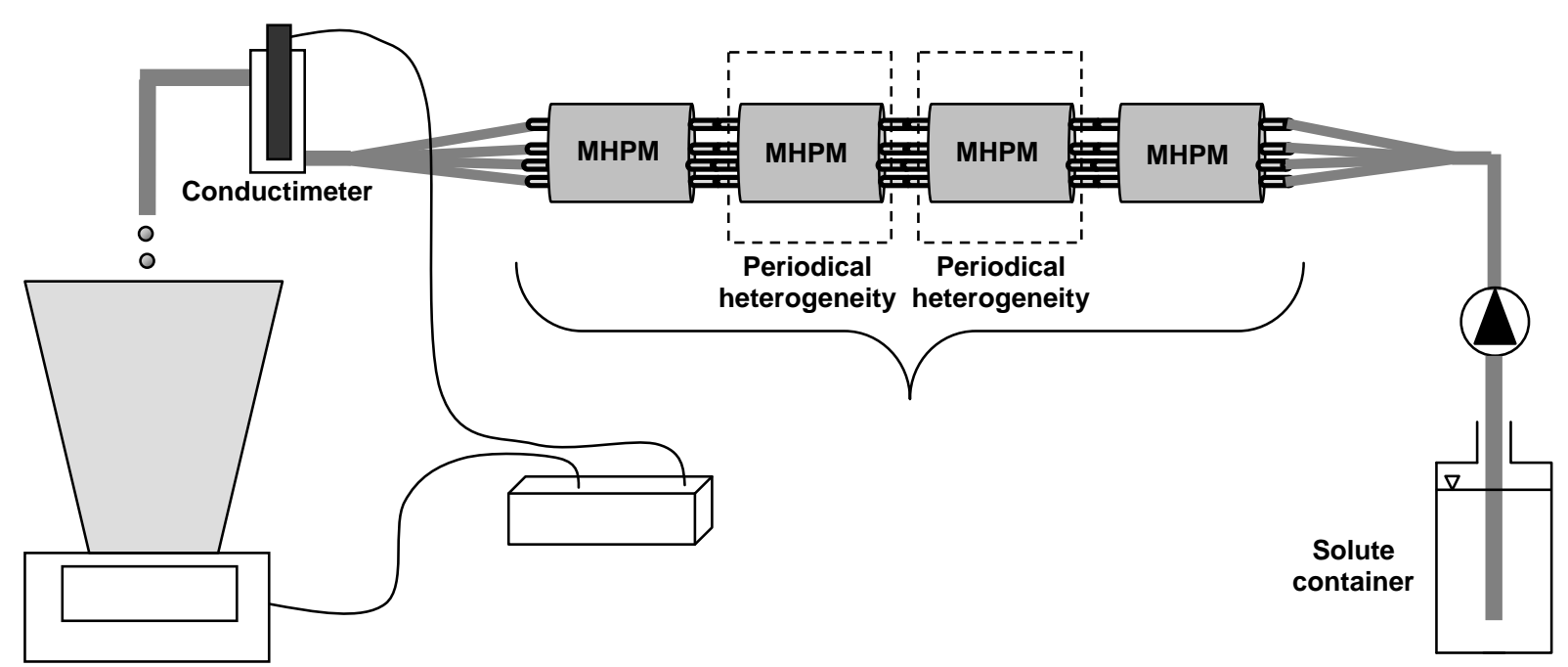

2

Figure 3. Experimental setup with an example of a series of $N=4$ columns. Only the in-between column correspond to periodical heterogeneities. 

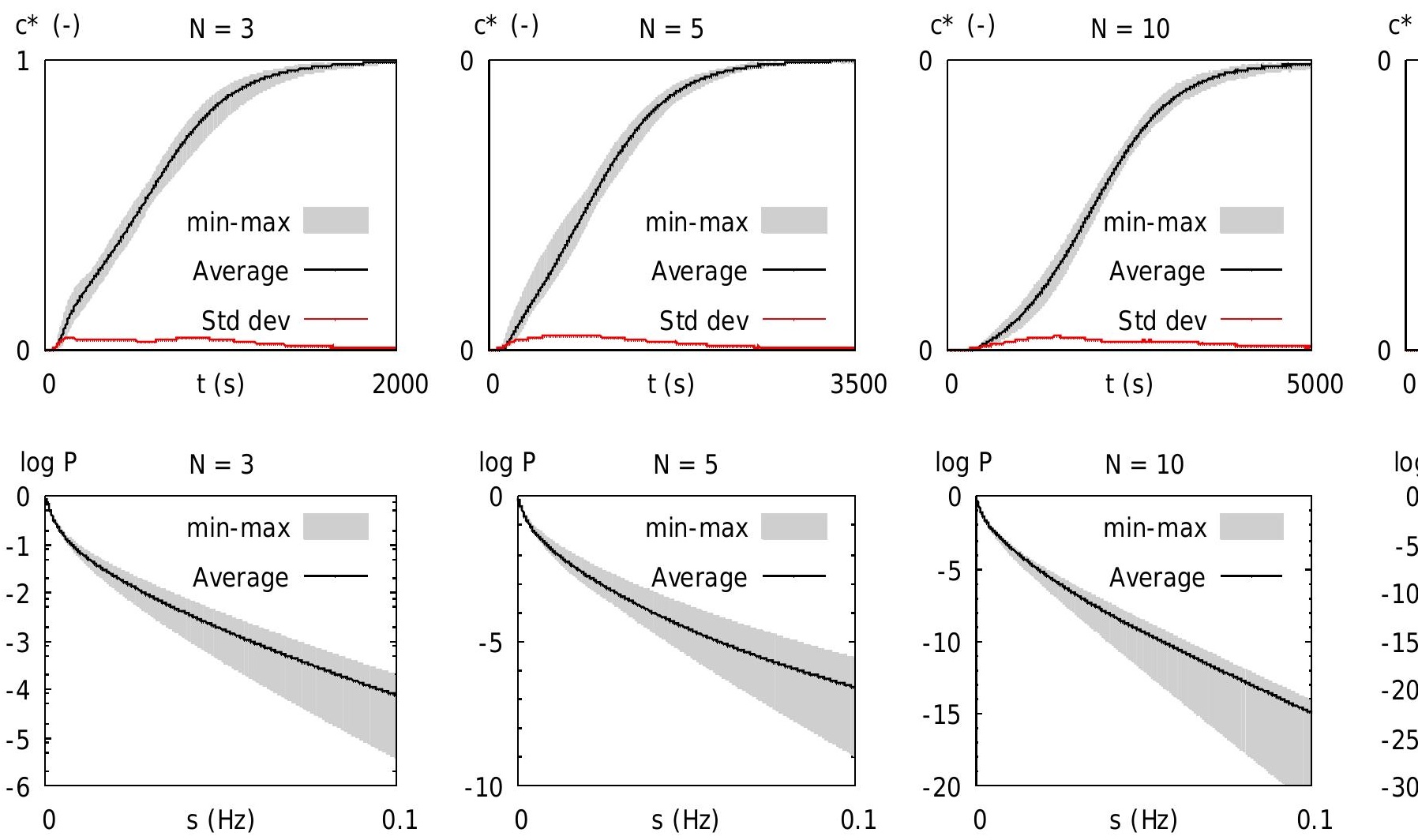

Figure 4. Breakthrough curves and the Laplace transforms of their derivatives for $N=3,5,10$, and 15 MHPM. Note the logarithmic coordinates for the Laplace transform. 

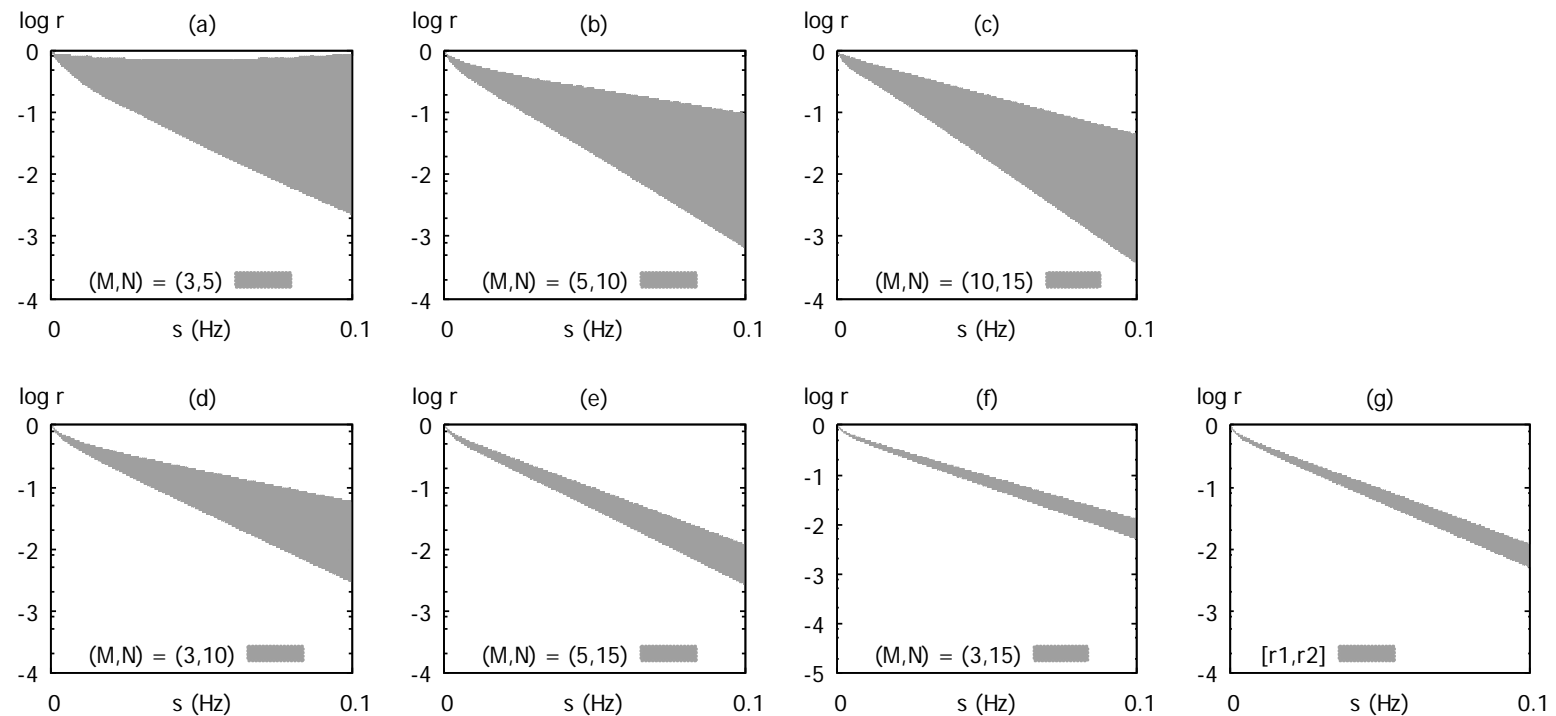

2

Figure 5. Unit response of a column inferred from the Laplace transforms of the experimental signals. 

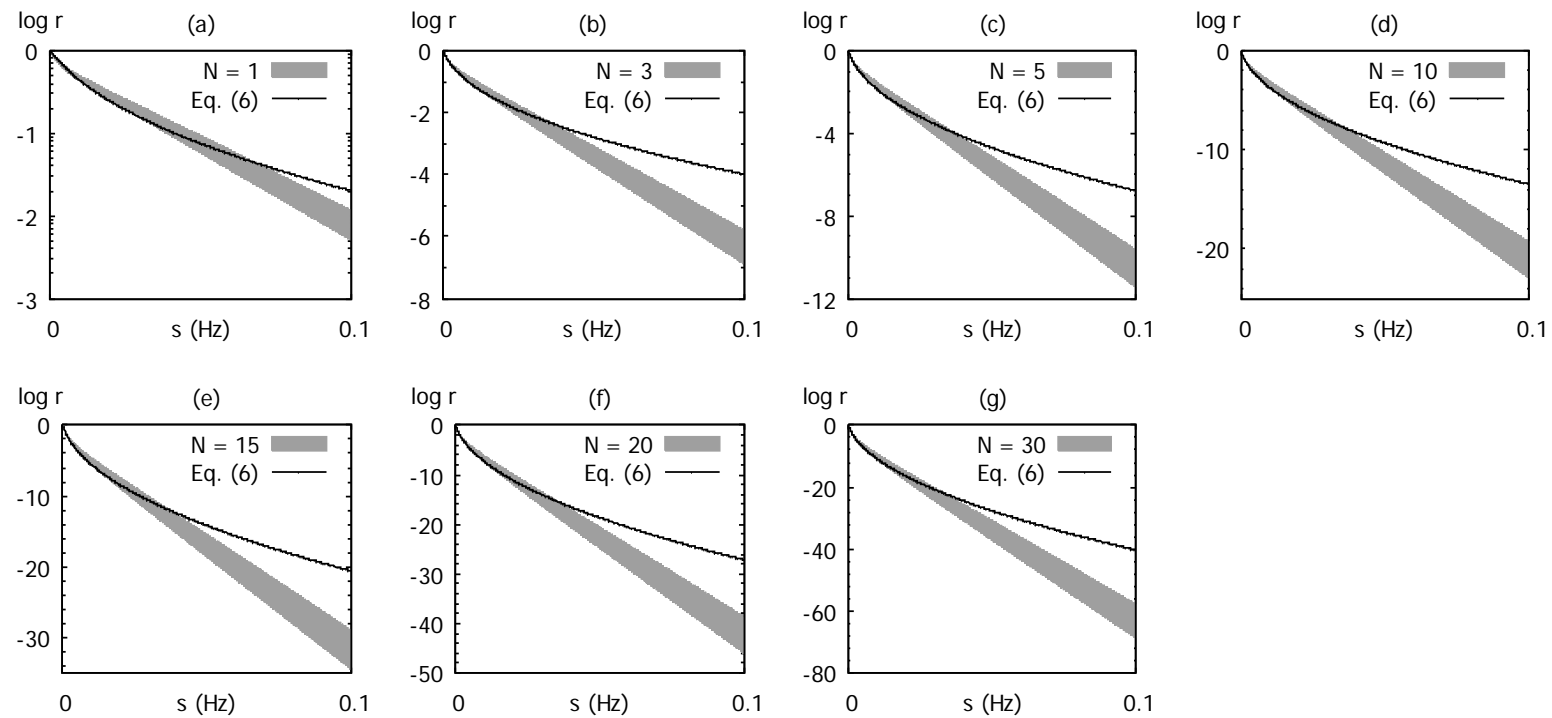

Figure 6. Best AD model fits in the Laplace space for various of $N$. 